\title{
Dynamic 4D MRI for Characterization of Parathyroid Adenomas: Multiparametric Analysis
}

\author{
D. K. Nael, J. Hur, A. Bauer, R. Khan, A. Sepahdari, R. Inampudi, and M. Guerrero
}

\begin{abstract}
BACKGROUND AND PURPOSE: The hypervascular nature of parathyroid adenomas can be explored by proper dynamic imaging to narrow the target lesions for surgical exploration. The purpose of this study was to establish MR perfusion characteristics of parathyroid adenomas to differentiate them from their mimics, such as subjacent thyroid tissue and cervical lymph nodes.
\end{abstract}

MATERIALS AND METHODS: Preoperative high-spatial and -temporal resolution dynamic 4D contrast-enhanced MR imaging in 30 patients with surgically proved parathyroid adenomas was evaluated retrospectively. Using coregistered images, we placed ROIs over the parathyroid adenoma, thyroid gland, and a cervical lymph node (jugulodigastric) to obtain peak enhancement, time-to-peak, wash-in, and washout in each patient. Data were analyzed by logistic regression and analysis of variance. Receiver operating characteristic analysis was performed to determine the optimal parameters for determination of parathyroid adenomas versus thyroid tissue and cervical lymph nodes.

RESULTS: Parathyroid adenomas showed significantly $(P<.05)$ faster time-to-peak, higher wash-in, and higher washout compared with cervical lymph nodes and significantly $(P<.05)$ higher peak enhancement, faster time-to-peak, higher wash-in, and higher washout compared with thyroid tissue. Logistic regression analysis indicated significant contribution from time-to-peak $(P=.02)$, wash-in $(P=.03)$, and washout $(P=.008)$ for differentiation of parathyroid adenomas from thyroid and cervical lymph nodes. Using receiver operating characteristic analysis, we obtained the best diagnostic accuracy from a combination of time-to-peak/wash-in/washout in the differentiation of parathyroid adenomas versus lymph nodes (area under the curve, 0.96; sensitivity/specificity, 88\%/90\%) and in distinguishing parathyroid adenomas versus thyroid tissue (area under the curve, 0.96; sensitivity/specificity, 91\%/95\%).

CONCLUSIONS: Dynamic 4D contrast-enhanced MR imaging can be used to exploit the hypervascular nature of parathyroid adenomas. Multiparametric MR perfusion can distinguish parathyroid adenomas from subjacent thyroid tissue or lymph nodes with diagnostic accuracies of $96 \%$.

ABBREVIATIONS: $\mathrm{AUC}=$ area under the curve; CAIPIRINHA = controlled aliasing in parallel imaging results in higher acceleration; PTA = parathyroid adenoma; ROC = receiver operating characteristic; TTP $=$ time-to-peak; TWIST $=$ time-resolved imaging with stochastic trajectories

S ingle parathyroid adenoma (PTA) is the most common cause of primary hyperparathyroidism, accounting for approximately $80 \%-90 \%$ of all cases. ${ }^{1}$ Definitive treatment requires surgical excision, and preoperative localization with imaging is com-

Received February 13, 2015; accepted after revision April 12.

From the Departments of Medical Imaging (K.N., J.H., A.B., R.K., R.I.) and Surgery (M.G.), University of Arizona, Tucson, Arizona; Department of Radiology (K.N.), Neuroradiology Section, Icahn School of Medicine at Mount Sinai, New York, New York; and Department of Radiological Sciences (A.S.), University of California, Los Angeles, Los Angeles, California.

Please address correspondence to Kambiz Nael, MD, Department of Radiology, Neuroradiology Section, Mount Sinai Hospital, 1176 5th Ave, Box 1235, New York, NY 10029; e-mail: Kambiznael@gmail.com; @kambiznael

-- Indicates open access to non-subscribers at www.ajnr.org

三 Indicates article with supplemental on-line table.

http://dx.doi.org/10.3174/ajnr.A4425 monly used to decrease the size of surgical incisions and complication rates. ${ }^{2}$

Imaging has been increasingly used for preoperative detection of parathyroid adenomas. While sonography and technetium Tc99m sestamibi scintigraphy have often been used as first-line imaging to localize PTA, these tests are often inconclusive. This situation has led to the development of multiphasic CT (4D CT), which identifies PTAs through their hypervascular perfusion pattern compared with lymph nodes and the thyroid gland. 4D CT has shown superior accuracy compared with scintigraphy, ${ }^{3}$ though the radiation dose remains as high as $5.56-10.4 \mathrm{mSv}^{4-6}$

MR imaging is an attractive alternative to both scintigraphy and $4 \mathrm{D}$ CT due to the lack of radiation and has been used for the evaluation of PTAs with some success, ${ }^{7-9}$ though not with 
the same effectiveness as 4D CT. Traditional technical limitations to localizing PTAs with MR imaging have recently been addressed with modern MR imaging technology. These include limited spatial and temporal resolution for multiphase dynamic contrast-enhanced MR imaging over a large FOV required for parathyroid imaging. This limitation can be addressed by the use of fast imaging tools such as time-resolved imaging with stochastic trajectories (TWIST) ${ }^{10}$ and improved parallel imaging techniques such as controlled aliasing in parallel imaging results in higher acceleration (CAIPIRINHA). ${ }^{11}$ The second limitation is inhomogeneity of fat suppression in the neck required for detection of small parathyroid adenomas. The Dixon fat-suppression technique ${ }^{12}$ can dramatically improve this shortcoming. ${ }^{13}$

In this study by using a modified dynamic contrast-enhanced sequence with incorporation of a TWIST-CAIPIRINHA combination and the Dixon fat saturation technique, we sought to describe MR perfusion characteristics of PTAs in a cohort of patients with pathology-proved PTA. We hypothesized that MR perfusion biomarkers can differentiate PTA from PTA mimics, such as subjacent thyroid gland and cervical lymph nodes.

\section{MATERIALS AND METHODS}

Informed consent was waived by the institutional review board for this retrospective single-institution study. Inclusion criteria were the following: 1) a patient with a known single PTA (confirmed by surgical pathology); and 2) preoperative dynamic 4D contrast-enhanced MR imaging available. Preoperative serum $\mathrm{Ca}^{2+}$ and parathyroid hormone levels were also documented for each patient.

\section{Imaging Protocol}

All patients underwent MR imaging on a 3T Skyra MR imaging system (Siemens, Erlangen, Germany). A combination of 12-element head and neck coil was used for radiofrequency signal reception. Dynamic 4D contrast-enhanced MR imaging was performed by using a $3 \mathrm{D}$ volumetric interpolated examination sequence with the following parameters: $\mathrm{TR}=4.06 \mathrm{~ms}$, first $\mathrm{TE}=$ $1.31 \mathrm{~ms}$, second $\mathrm{TE}=2.54 \mathrm{~ms}$, flip angle $=9^{\circ}$, matrix $=160 \mathrm{~mm}$, FOV $=200 \mathrm{~mm}, 60$ sections $\times 2 \mathrm{~mm}$ thick. To improve inhomogeneity of fat suppression in the neck, we used the Dixon fatsuppression technique as described in prior reports. ${ }^{12,13}$ The TWIST view-sharing and Dixon fat/water separation were merged into 1 pulse sequence. ${ }^{14}$ Bipolar readout gradients were used to produce 2 partial echoes at the first in-phase $(\mathrm{TE}=1.31 \mathrm{~ms})$ and the second opposed-phase ( $\mathrm{TE}=2.54 \mathrm{~ms}$ ). Bipolar gradients allowed a shorter TR and less echo asymmetry. Integration of TWIST as an echo-sharing technique with a sampling density of $33 \%$ resulted in $\times 2$ acceleration. In addition, CAIPIRINHA with an acceleration factor of 4 was incorporated as a parallelacquisition technique. This combination resulted in a net acceleration of 8 , which was used to acquire a $3 \mathrm{D}$ dataset with a voxel size of $1.3 \times 1.3 \times 2 \mathrm{~mm}^{3}$ and a temporal resolution of 6 seconds over a craniocaudal coverage of $120 \mathrm{~mm}$, spanning the inferior mandibular rim to the carina. Twenty-four temporal frames were obtained during 140 seconds of acquisition time, 4

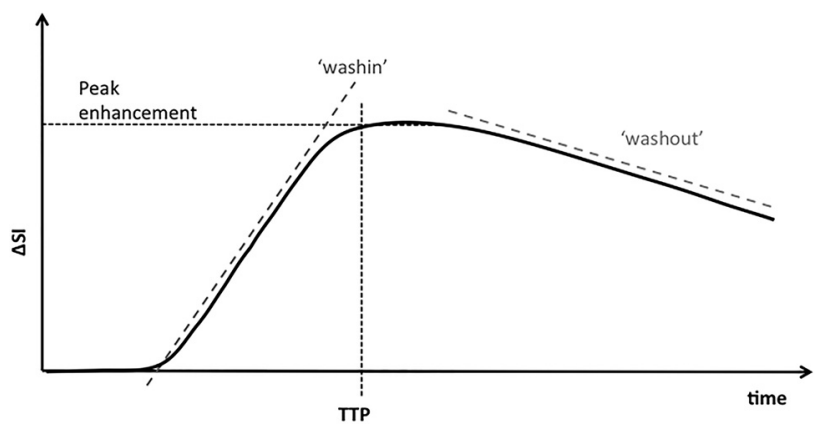

FIG 1. Concentration-time curve. Peak enhancement: maximal concentration of contrast agent with time: Peak = max $C(t)$. Time-topeak enhancement: the time needed for the contrast agent to reach its maximum concentration: TTP $=\arg \max C(t)$. Wash-in: initial upslope of the concentration-time curve (slope from the end of the baseline to the peak of the curve). Washout: down-slope of the concentration time curve (negative slope from the peak to the last acquisition time point). $\Delta \mathrm{SI}$ indicates change in signal intensity.

before contrast injection to establish a baseline. A total of 0.1 $\mathrm{mmol} / \mathrm{kg}$ of gadolinium was injected at $4 \mathrm{~mL} / \mathrm{s}$.

\section{Image Analysis}

The perfusion datasets were processed by using commercially available US Food and Drug Administration-approved software (Olea Sphere; Olea Medical SAS, La Ciotat, France). Motion correction was implemented automatically by the software. Signalto-concentration conversion was performed $[C(t)=S(t)-S O]$, with $S O$ being the baseline signal. The arterial input function was selected automatically to deconvolute the concentration-time curve, assuming a multicompartmental model. ${ }^{15}$ The concentration-time curve was then reconstructed from this analysis to obtain a noise-free signal.

Multiparametric quantitative perfusion parameters, including peak enhancement, time-to-peak (TTP), wash-in, and washout, were then computed from the reconvolved concentration-time curve. The wash-in and washout were defined by the initial upslope and downslope of the concentration-time curve, respectively. The definition of these parameters and how they are calculated from the concentration time curves are shown in Fig 1.

Image coregistration was performed by using a 6- $d f$ transformation and a mutual-information cost function. This was followed by visual inspection to ensure adequate alignment. PTAs were identified by a fellowship-trained neuroradiologist in conjunction with the endocrine surgeon who performed all the surgeries. Using coregistered images, we placed ROIs over the known PTA, thyroid gland, and cervical lymph node (jugulodigastric station). Using the same ROIs, we also calculated the percentage of internal fat content of each PTA on precontrast T1 images before and after Dixon fat saturation.

\section{Statistical Analysis}

Statistical analysis was performed by using MedCalc for Windows, Version 14.12.0 (MedCalc Software, Mariakerke, Belgium). The semiquantitative data, including the peak enhancement, TTP, wash-in, and washout values of the PTA, thyroid gland, and cervical lymph node were plotted as mean and SD and tested for 
Table 1: Multivariate analysis of MR perfusion parameters for PTA, thyroid gland, and cervical lymph node ${ }^{a}$

\begin{tabular}{|c|c|c|c|c|}
\hline & PTA & Thyroid Gland & Lymph Node & ANOVA ( $P$ Value) \\
\hline Peak enhancement, $\mathrm{AU}$ & $360.9 \pm 87$ & $214 \pm 47$ & $305.3 \pm 153$ & $\begin{array}{l}\text { PTA vs thyroid: }<.001^{6} \\
\text { PTA vs LN: } .38\end{array}$ \\
\hline Time-to-peak (seconds) & $35.2 \pm 12.8$ & $48.7 \pm 22.6$ & $64.4 \pm 35$ & $\begin{array}{l}\text { PTA vs thyroid: } .001^{\mathrm{b}} \\
\text { PTA vs LN: }<.001^{\mathrm{b}}\end{array}$ \\
\hline Wash-in, AU & $7.5 \pm 2.6$ & $3.9 \pm 1.2$ & $4.4 \pm 1.9$ & $\begin{array}{l}\text { PTA vs thyroid: }<.001^{\mathrm{b}} \\
\text { PTA vs LN: }<.001^{\mathrm{b}}\end{array}$ \\
\hline Washout, AU & $0.80 \pm 0.24$ & $0.43 \pm 0.17$ & $0.58 \pm 0.31$ & $\begin{array}{l}\text { PTA vs thyroid: }<.001^{\mathrm{b}} \\
\text { PTA vs LN: } .016^{\mathrm{b}}\end{array}$ \\
\hline
\end{tabular}

Note:- LN indicates lymph node; AU, arbitrary units.

${ }^{a}$ Data are means $\pm \mathrm{SD}$.

b Significant.
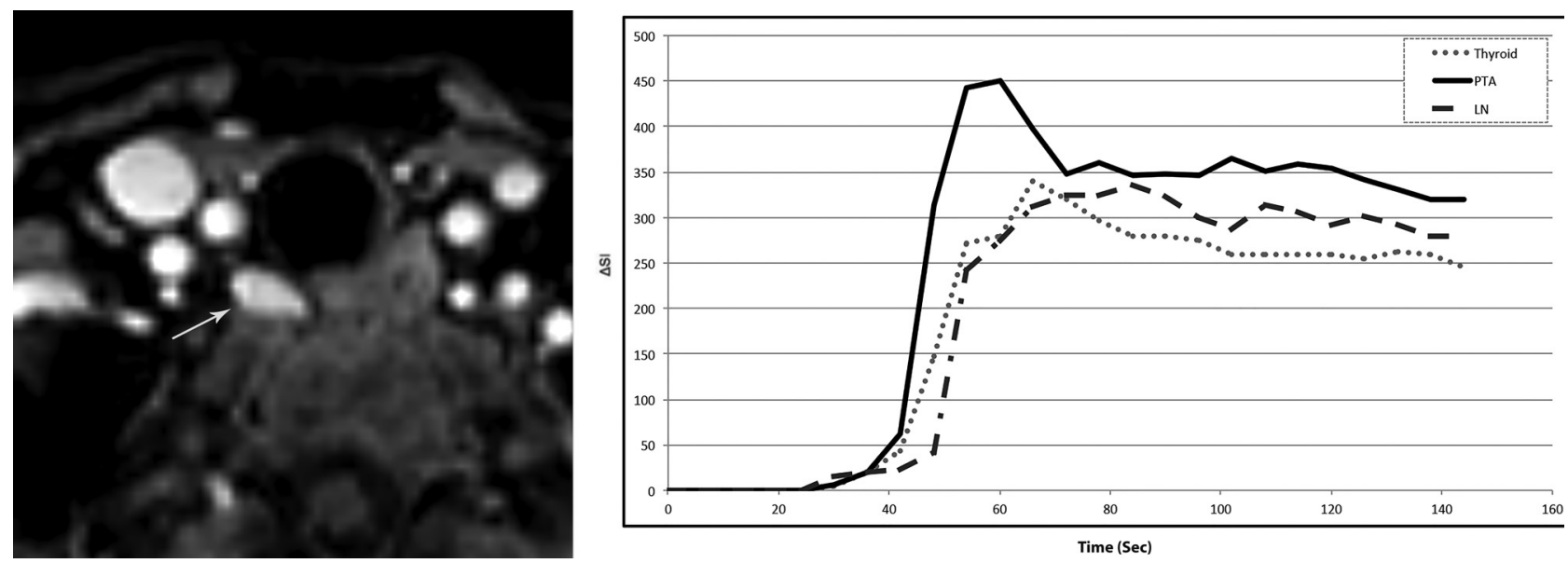

FIG 2. A 68-year-old woman with primary hyperparathyroidism (parathyroid hormone $=120 \mathrm{pg} / \mathrm{mL}, \mathrm{Ca}^{2+}=11.2 \mathrm{mg} / \mathrm{dL}$ ). Axial arterial phase contrast-enhanced image from MR perfusion demonstrates a PTA (arrow) in the right tracheoesophageal groove. Contrast-time curve analysis from ROls placed over the PTA (arrow), thyroid gland, and a jugulodigastric lymph node shows significantly faster TTP and higher wash-in and washout values in the PTA compared with the thyroid gland and cervical lymph node. PTA: TTP, 37 seconds; wash-in, 7.8; washout, 0.58 . Thyroid: TTP, 42 seconds; wash-in, 5.4 washout, 0.46. Lymph node: TTP, 60 seconds; wash-in, 4.8; washout, 0.29.

statistical significance by using a repeated-measures ANOVA with protected least-squares-difference post hoc tests. Logistic regression analysis was performed to identify the contribution of each perfusion parameter to the model. Receiver operating characteristic (ROC) analysis was performed to ascertain the optimal parameters and threshold for determination of PTA versus cervical lymph node and thyroid gland.

We calculated optimal thresholds for each ROC curve to maximize both sensitivity and specificity using the Youden statistic. Subsequently, an ROC curve for the combination of parameters was calculated, extrapolating from the maximum-likelihoodestimation model of combining classifiers. ${ }^{16}$ The area under the curve was calculated for the ROC curve of each individual classifier and for the combined ROC curves. Correlation coefficients of perfusion parameters in PTA were calculated against the internal fat content of the PTA with $95 \%$ confidence intervals. The significance level was defined as $P<.05$.

\section{RESULTS}

Thirty patients (20 women, 10 men) with a mean age of 60 years (range, 27-79 years) were included. The preoperative serum $\mathrm{Ca}^{2+}$ ranged from 9.9 to $12 \mathrm{mg} / \mathrm{dL}$ (mean, $10.92 \mathrm{mg} / \mathrm{dL}$; interquartile range, $10.6-11.2 \mathrm{mg} / \mathrm{dL}$ ). The preoperative serum parathyroid hormone ranged from 52 to $246 \mathrm{pg} / \mathrm{mL}$ (mean, $115.3 \mathrm{pg} / \mathrm{mL}$; interquartile range, $78-147 \mathrm{pg} / \mathrm{mL}$ ). The percentage of fat content of PTAs ranged from $7.2 \%$ to $71 \%$ (mean, $38.7 \%$; interquartile range, $21.3 \%-59.5 \%)$. Patient demographic data and the size, weight, and location of parathyroid adenomas are listed in the On-line Table.

Despite using motion correction, we found that in 7 patients (23\%), 1-2 temporal frames were affected by motion artifacts. These frames were excluded from perfusion analysis. The mean \pm SD of MR perfusion parameters for PTA, thyroid gland, and cervical lymph node and the corresponding $P$ values by using ANOVA analysis are summarized in Table 1.

\section{PTA versus Cervical Lymph Node}

Three perfusion parameters, including TTP, wash-in, and washout, were significantly different between PTAs and cervical lymph nodes (Table 1). PTAs showed significantly faster arterial enhancement with a mean TTP enhancement of 29 seconds earlier than that in lymph nodes $(P<.001)$. PTAs also showed significantly higher $(P<.001)$ wash-in and higher $(P<.001)$ washout values in comparison with lymph nodes (Fig 2$)$. The peak enhancement was not significantly different between PTAs and cervical lymph nodes $(P=.38)$.

ROC curve analyses for differentiation of PTAs versus cervical lymph nodes and PTAs versus thyroid glands are shown in Fig 3. Optimal threshold values, area under the curve (AUC), and corresponding sensitivity and specificity for each perfusion parameter for the differentiation of PTA versus lymph node are summarized in Table 2. With multiparametric 

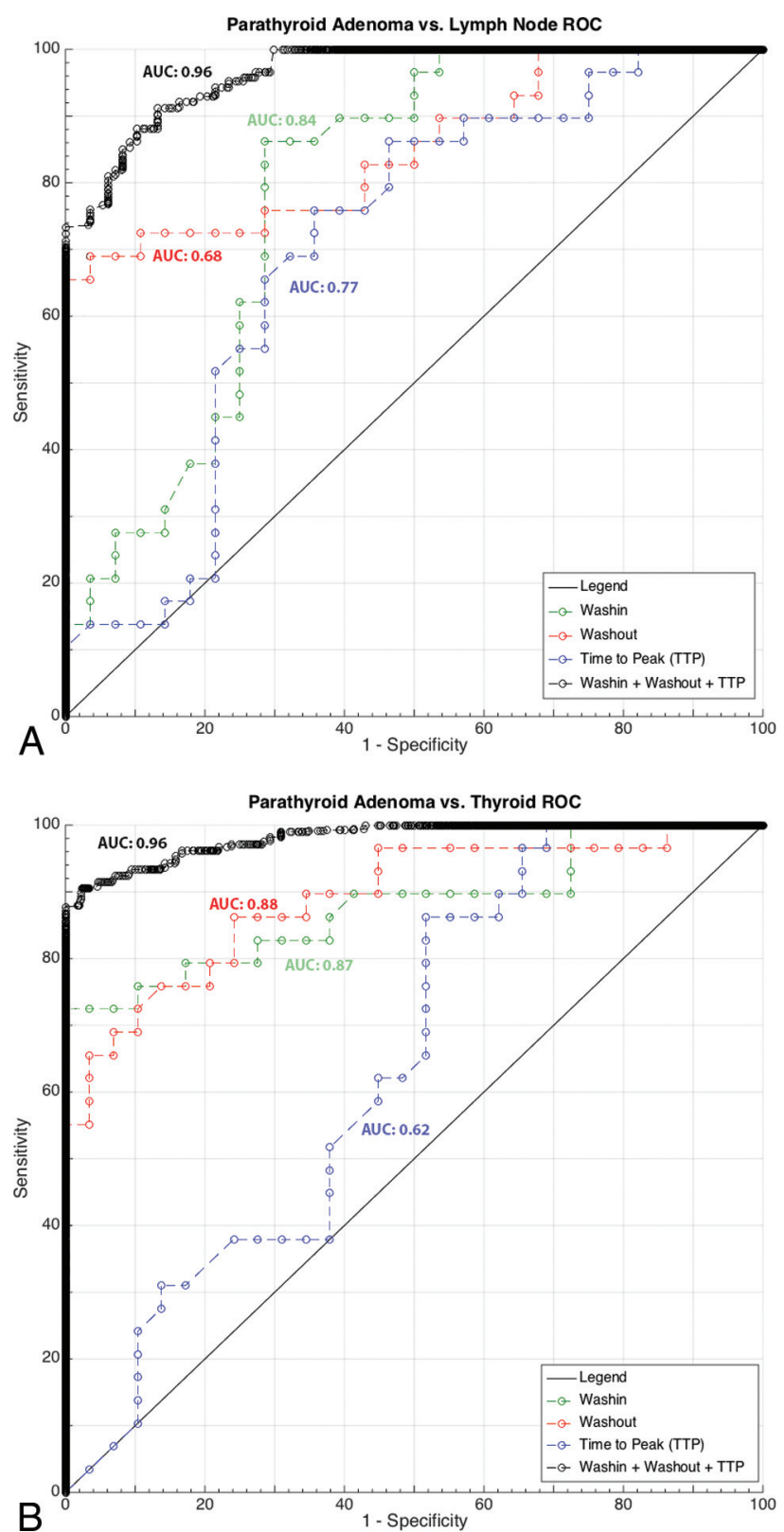

FIG 3. ROC analysis with the AUC for each imaging biomarker and best combined AUC for PTA-versus-lymph node and PTA-versus-thyroid gland is shown.

MR perfusion analysis, a combination of TTP (threshold of $<40$ seconds), wash-in (threshold of $>6.3$ ), and washout (threshold of $>0.62$ ) improved the diagnostic power, resulting in an AUC of 0.96 with sensitivity/specificity of $88 \% / 90 \%$ for differentiating PTAs (Fig 3).

\section{PTA versus Thyroid Gland}

All 4 perfusion parameters were significantly different between PTAs and thyroid glands (Table 1). Again PTAs showed significantly faster arterial enhancement with a mean TTP enhancement difference of 13 seconds earlier than that in thyroid glands $(P=.001)$. There was significantly higher peak enhancement $(P<.001)$, wash-in $(P<.001)$, and washout $(P=.016)$ in PTAs compared with thyroid glands (Fig 4).

Optimal threshold values, AUC, and corresponding sensitivity and specificity for each perfusion parameter for the differentiation of PTA versus thyroid gland are summarized in Table 2. The best sensitivity ( $86 \%$ ) to differentiate PTA from thyroid gland was obtained by using the TTP value at a threshold of $>37$ seconds. Wash-in showed the highest specificity (90\%) for differentiating PTA versus thyroid gland at a threshold value of $>5.27$. With multiparametric MR perfusion analysis, a combination of TTP (threshold of $>30$ seconds), wash-in (threshold of $>5.86$ ), and washout (threshold of $>0.67$ ) improved the diagnostic power resulting in an AUC of 0.96 with sensitivity/specificity of 91\%/95\% (Fig 3).

Logistic regression analysis indicated significant contribution from TTP $(P=.02 ; 95 \%$ CI, 0.88-.99), wash-in $(P=.03 ; 95 \% \mathrm{CI}$, $1.04-3.06)$, and washout $(P=.008$; 95\% CI, 3.28-37.15) for the differentiation of PTA from thyroid and cervical lymph nodes. There was no contribution from peak enhancement to differentiate PTA from thyroid and cervical lymph nodes $(P=.56$; 95\% CI, $0.98-1.00)$.

Correlation analysis between the percentage of internal fat content and multiparametric perfusion values of PTAs showed only a significant $(P=.019)$ negative correlation $(r=-0.632)$ with washout values of PTAs. The correlation coefficients between the percentage of internal fat content of PTAs with peak enhancement, time-to-peak, and wash-in values were 0.150 , 0.004 , and -0.007 , respectively.

\section{DISCUSSION}

In this study by using a modified MR imaging sequence with incorporation of fast imaging tools, for the first time, we describe MR perfusion characteristics of PTAs. We performed a multiparametric quantitative analysis and showed significant differences between perfusion biomarkers in PTAs versus cervical lymph nodes and thyroid gland. We note 3 findings:

First, the described MR imaging technique has significantly improved the traditional drawbacks of MR imaging, including limited spatial and temporal resolution. Using a modified MR image that incorporates fast imaging tools such as TWIST $^{10}$ and CAIPIRINHA $^{11}$ has allowed acquisition of multiphase contrastenhanced MR imaging with high spatial $\left(1.3 \times 1.3 \times 2 \mathrm{~mm}^{3}\right)$ and temporal (6 seconds) resolution.

Fast image acquisition and high spatial resolution have long been major advantages of CT; hence, significant attention has been given to $4 \mathrm{D}$ CT for the detection of PTAs. However, the main disadvantage of CT that can be addressed by the described MR imaging technique is radiation.

There is significant ongoing discussion in the literature as to which 4D CT protocol has a better diagnostic accuracy, whether a noncontrast scan is required, and how many postcontrast phases are required for optimal results without the added and unnecessary radiation dose. This discussion is beyond the scope of this article; however, effective radiation doses ranging from 5.56 to $10.4 \mathrm{mSv}^{4-6}$ have been reported, depending on the acquisition scheme used.

Our second finding is that the described MR perfusion technique can successfully exploit the hypervascular nature of PTAs, a feature that can be used to differentiate them from PTA candidates such as subjacent cervical lymph node and thyroid tissue. 
Table 2: ROC analysis of multiparametric MR perfusion for differentiation of PTA from cervical lymph nodes and thyroid gland

\begin{tabular}{|c|c|c|c|c|c|c|c|c|}
\hline & \multicolumn{4}{|c|}{ PTA vs Lymph Node } & \multicolumn{4}{|c|}{ PTA vs Thyroid Gland } \\
\hline & AUC & Threshold & Sensitivity & Specificity & AUC & Threshold & Sensitivity & Specificity \\
\hline Peak enhancement & 0.62 & $>340$ & $65 \%$ & $64 \%$ & 0.94 & $>270$ & $83 \%$ & $93 \%$ \\
\hline Time-to-peak (seconds) & 0.77 & $<49$ & $66 \%$ & $71 \%$ & 0.62 & $<37$ & $86 \%$ & $48 \%$ \\
\hline Wash-in & 0.84 & $>5.52$ & $72 \%$ & $89 \%$ & 0.87 & $>5.27$ & $76 \%$ & $90 \%$ \\
\hline Washout & 0.68 & $>0.62$ & $76 \%$ & $64 \%$ & 0.88 & $>0.63$ & $76 \%$ & $86 \%$ \\
\hline
\end{tabular}
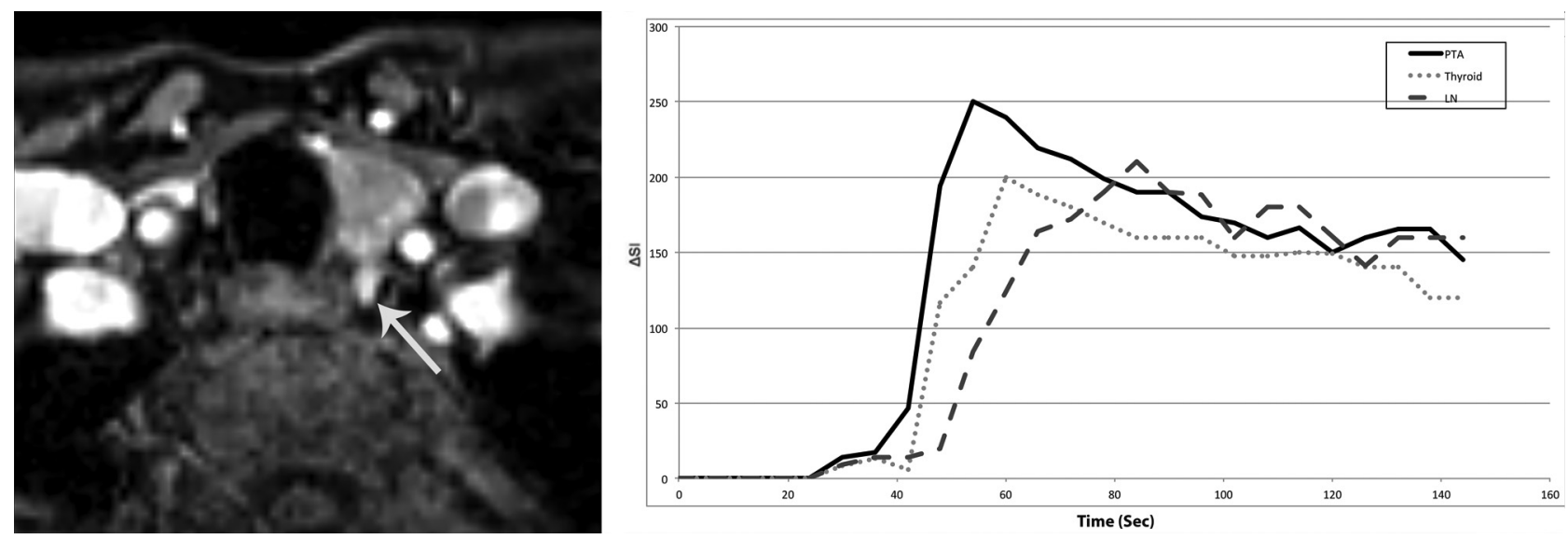

FIG 4. A 47-year-old woman with primary hyperparathyroidism (parathyroid hormone $=164 \mathrm{pg} / \mathrm{mL}, \mathrm{Ca}^{2+}=10.8 \mathrm{mg} / \mathrm{dL}$ ). Axial arterial phase contrast-enhanced image from MR perfusion data demonstrates a PTA (arrow) in the left tracheoesophageal groove. Contrast-time curve analysis from ROIs placed over the PTA, thyroid gland, and a cervical lymph node shows significantly faster TTP and higher wash-in and significant washout values in the PTA compared with the thyroid gland and cervical lymph node. PTA: TTP, 30 seconds; wash-in, 5.6; washout, 0.64 Thyroid: TTP, 38 seconds; wash-in, 3.8 seconds; washout, 0.43 seconds. Lymph node: TTP, 62 seconds; wash-in, 2.9; washout, 0.23.

The hypervascular nature of PTAs was shown in the 1970s by the use of arterial conventional angiography, demonstrating arterial blush as an indication of PTA against normal thyroid and parathyroid glands. ${ }^{17,18}$ Similar principles have been applied to dynamic contrast-enhanced CT and can be applied to MR imaging as shown in our results for the detection of PTAs.

Because continuous CT acquisition during the entire dynamic course of contrast through the parathyroid glands is restricted due to radiation concerns, 4D CT provides only snapshots of contrast dynamics at certain time points (depending on the number of acquisitions). There is no consensus on the number and timeinterval between CT acquisitions for an optimal 4D CT.

Therefore investigators have tried to identify acquisition schemes by using a combination of unenhanced and multiple postcontrast phases including 2 phases, ${ }^{19-21} 2.5$ phases, ${ }^{22,23} 3$ phases, ${ }^{4,24}$ or 4 phases, ${ }^{25-27}$ with the time interval ranging from 30 to 90 seconds, each with strengths and limitations.

Compared with CT, the unique ability of MR imaging to sample many points on the contrast-versus-time curve allowed us to obtain perfusion characteristics such as TTP, peak-enhancement, wash-in, and washout. In our study, TTP and wash-in, 2 characteristics of arterial enhancement, were significantly higher in PTAs, indicative of their hypervascular nature in comparison with cervical lymph nodes and thyroid tissue. PTAs showed significant early arterial enhancement with a mean TTP of 13 and 29 seconds earlier than thyroid tissue and normal cervical lymph nodes, respectively. Logistic regression analysis indicated significant contribution from TTP, wash-in, and washout for the differentiation of PTA from thyroid and cervical lymph nodes but not from peak enhancement.
In addition, we showed the added value of multiparametric quantitative perfusion analysis to improve diagnostic accuracy over a single perfusion classifier to differentiate PTA among PTA candidates such as subjacent thyroid gland and cervical lymph nodes. Using multiparametric MR perfusion and combined ROC analysis, we found that the best overall model to distinguish PTA from cervical lymph node or subjacent thyroid tissue consisted of a combination of TTP, wash-in, and washout, yielding an AUC of 0.96 , superior to any individual or combination of other classifiers.

Finally, we found a significant $(P=.019)$ negative correlation $(r=-0.632)$ between fat content percentage and washout values of PTAs. The clinical significance of this observation is rather unclear at this point. We assume that while TTP and wash-in are indicators of hypervascularity, the washout may be a reflection of the cellular content of PTAs. In other words, there is delayed clearance and contrast washout in lipid-rich adenomas with less cellularity. This finding should be interpreted with caution and within the 2-minute imaging window used in our study because some of these adenomas may further washout later, outside our imaging window.

This study has several limitations, including a relatively small sample size drawn from a single institution, possibly introducing a sample bias. In addition, inclusion of patients with known PTAs can further introduce selection bias. Another limitation is the retrospective nature of the study, possibly introducing unknown bias. The diagnostic accuracy of this MR imaging technique for the localization of PTA should be evaluated prospectively in a larger cohort. The last limitation is the insufficient availability and technical demands due to the 3T MR imaging scanners and mul- 
ticoil technology required for parallel imaging and the echo-sharing technique. Although all of these technologies are now commercially available, broad accessibility across imaging centers is still limited.

\section{CONCLUSIONS}

Dynamic 4D contrast-enhanced MR imaging can be obtained and used to exploit the hypervascular nature of PTAs. Multiparametric MR perfusion can distinguish PTAs from subjacent thyroid tissue or lymph nodes with a diagnostic accuracy of $96 \%$.

\section{ACKNOWLEDGMENTS}

We thank Elizabeth A. Krupinski, PhD, for help with statistical analysis.

Disclosures: Kambiz Nael—UNRELATED: Consultancy: Olea Medical. * *Money paid to the institution.

\section{REFERENCES}

1. Heath H 3rd, Hodgson SF, Kennedy MA. Primary hyperparathyroidism: incidence, morbidity, and potential economic impact in a community. N Engl J Med 1980;302:189-93 CrossRef Medline

2. Chen H, Mack E, Starling JR. A comprehensive evaluation of perioperative adjuncts during minimally invasive parathyroidectomy: which is most reliable? Ann Surg 2005;242:375-80; discussion 380-83 Medline

3. Mortenson MM, Evans DB, Lee JE, et al. Parathyroid exploration in the reoperative neck: improved preoperative localization with 4Dcomputed tomography. J Am Coll Surg 2008;206:888 -95; discussion 895-96 CrossRef Medline

4. Kelly HR, Hamberg LM, Hunter GJ. 4D-CT for preoperative localization of abnormal parathyroid glands in patients with hyperparathyroidism: accuracy and ability to stratify patients by unilateral versus bilateral disease in surgery-naive and re-exploration patients. AJNR Am J Neuroradiol 2014;35:176-81 CrossRef Medline

5. Mahajan A, Starker LF, Ghita M, et al. Parathyroid four-dimensional computed tomography: evaluation of radiation dose exposure during preoperative localization of parathyroid tumors in primary hyperparathyroidism. World J Surg 2012;36:1335-39 CrossRef Medline

6. Madorin CA, Owen R, Coakley B, et al. Comparison of radiation exposure and cost between dynamic computed tomography and sestamibi scintigraphy for preoperative localization of parathyroid lesions. JAMA Surg 2013;148:500-03 CrossRef Medline

7. Gotway MB, Reddy GP, Webb WR, et al. Comparison between MR imaging and 99mTc MIBI scintigraphy in the evaluation of recurrent of persistent hyperparathyroidism. Radiology 2001;218:783-90 CrossRef Medline

8. Lee VS, Spritzer CE, Coleman RE, et al. The complementary roles of fast spin-echo MR imaging and double-phase $99 \mathrm{~m}$ Tc-sestamibi scintigraphy for localization of hyperfunctioning parathyroid glands. AJR Am J Roentgenol 1996;167:1555-62 CrossRef Medline

9. Grayev AM, Gentry LR, Hartman MJ, et al. Presurgical localization of parathyroid adenomas with magnetic resonance imaging at 3.0 $\mathrm{T}$ : an adjunct method to supplement traditional imaging. Ann Surg Oncol 2012;19:981-89 CrossRef Medline

10. Song T, Laine AF, Chen Q, et al. Optimal k-space sampling for dy- namic contrast-enhanced MRI with an application to MR renography. Magn Reson Med 2009;61:1242-48 CrossRef Medline

11. Breuer FA, Blaimer M, Heidemann RM, et al. Controlled aliasing in parallel imaging results in higher acceleration (CAIPIRINHA) for multi-slice imaging. Magn Reson Med 2005;53:684-91 CrossRef Medline

12. Dixon WT. Simple proton spectroscopic imaging. Radiology 1984; 153:189-94 CrossRef Medline

13. Barger AV, DeLone DR, Bernstein MA, et al. Fat signal suppression in head and neck imaging using fast spin-echo-IDEAL technique. AJNR Am J Neuroradiol 2006;27:1292-94 Medline

14. Le Y, Kroeker R, Kipfer HD, et al. Development and evaluation of TWIST Dixon for dynamic contrast-enhanced (DCE) MRI with improved acquisition efficiency and fat suppression. J Magn Reson Imaging 2012; 36:483-91 CrossRef Medline

15. Tofts PS. Modeling tracer kinetics in dynamic Gd-DTPA MR imaging. J Magn Reson Imaging 1997;7:91-101 CrossRef Medline

16. Haker S, Wells WM 3rd, Warfield SK, et al. Combining classifiers using their receiver operating characteristics and maximum likelihood estimation. Med Image Comput Comput Assist Interv 2005;8(pt 1):506-14 Medline

17. Doppman JL. Parathyroid localization: arteriography and venous sampling. Radiol Clin North Am 1976;14:163-88 Medline

18. Miller DL, Doppman JL. Parathyroid angiography. Ann Intern Med 1987;107:942-43 CrossRef Medline

19. Linda DD, Ng B, Rebello R, et al. The utility of multidetector computed tomography for detection of parathyroid disease in the setting of primary hyperparathyroidism. Can Assoc Radiol J 2012;63: 100-08 CrossRef Medline

20. Gafton AR, Glastonbury CM, Eastwood JD, et al. Parathyroid lesions: characterization with dual-phase arterial and venous enhanced CT of the neck. AJNR Am J Neuroradiol 2012;33:949-52 CrossRef Medline

21. Noureldine SI, Aygun N, Walden MJ, et al. Multiphase computed tomography for localization of parathyroid disease in patients with primary hyperparathyroidism: how many phases do we really need? Surgery 2014;156:1300-06; discussion 1306-07 CrossRef Medline

22. Kutler DI, Moquete R, Kazam E, et al. Parathyroid localization with modified 4D-computed tomography and ultrasonography for patients with primary hyperparathyroidism. Laryngoscope 2011;121: 1219-24 CrossRef Medline

23. Harari A, Zarnegar R, Lee J, et al. Computed tomography can guide focused exploration in select patients with primary hyperparathyroidism and negative sestamibi scanning. Surgery 2008;144:970-76; discussion 976-79 CrossRef Medline

24. Chazen JL, Gupta A, Dunning A, et al. Diagnostic accuracy of 4D-CT for parathyroid adenomas and hyperplasia. AJNR Am J Neuroradiol 2012;33:429-33 CrossRef Medline

25. Starker LF, Mahajan A, Björklund $\mathrm{P}$, et al. $4 \mathrm{D}$ parathyroid $\mathrm{CT}$ as the initial localization study for patients with de novo primary hyperparathyroidism. Ann Surg Oncol 2011;18:1723-28 CrossRef Medline

26. Beland MD, Mayo-Smith WW, Grand DJ, et al. Dynamic MDCT for localization of occult parathyroid adenomas in 26 patients with primary hyperparathyroidism. AJR Am J Roentgenol 2011;196:61-65 CrossRef Medline

27. Rodgers SE, Hunter GJ, Hamberg LM, et al. Improved preoperative planning for directed parathyroidectomy with 4-dimensional computed tomography. Surgery 2006;140:932-40; discussion 940-41 CrossRef Medline 